\title{
Conditioned taste aversion and unconditioned suppression of water intake induced by phenylpropanolamine in rats
}

\author{
PAUL J. WELLMAN \\ Texas A\&M University, College Station, Texas 77843 \\ and \\ PHILLIS B. MALPAS and KENNETH C. WIKLER \\ Bucknell University, Lewisburg, Pennsylvania 17837
}

\begin{abstract}
The putative aversive properties of phenylpropanolamine (PPA), an analog of amphetamine, were delineated in two behavioral tests. In a conditioned taste aversion paradigm, PPA $(10,20$, or $40 \mathrm{mg} / \mathrm{kg}$ ) was found to induce dose-dependent taste aversion, whereas identical dosages of PPA were found to produce dose-dependent unconditioned suppression of water intake in 23.5-h water-deprived rats. Comparison of the dose-response curves for the aversion and hypodipsia induced by PPA indicates that a single process, presumably malaise or toxicosis, may mediate these effects. These findings question the notion that PPA induces anorexia via activation of a CNS satiety mechanism and lend credence to the suggestion advanced herein that nonspecific malaise may mediate the moderate anorectic activity of PPA observed in rodents and humans.
\end{abstract}

Of the numerous drugs ascribed anorectic properties over the past four decades (Aviado, 1970; Costa \& Garratini, 1970), phenylpropanolamine (PPA) has perhaps held the greatest promise as a weight control substance for obese humans. Phenylpropanolamine, an analog of amphetamine, was reported to induce moderate anorexia and weight loss in rodents and humans but was without the properties of arousal and dependence that are characteristic of the amphetamines (Aviado, 1970; Epstein, 1959; Griboff, Berman, \& Silverman, 1975; Hoebel, 1976; Kornblith \& Hoebel, 1976). It is precisely because of these properties (and lack thereof) that over-the-counter weight loss preparations containing PPA have enjoyed considerable commercial success.

Although PPA is known to induce anorexia, the precise mechanism of this effect is unclear. A major problem in the interpretation of the anorexia induced by drugs such as PPA derives from the notion that all or a portion of the anorexia may result not from the induction of satiety, but rather from some malaiseinducing effect such as hyperthermia, gastrointestinal irritation, or arousal (Carey, 1979; Carlton, 1963; Wellman \& Peters, 1980). With regard to the possible side effects of PPA, Epstein (1959) and Kornblith and Hoebel (1976) reported that PPA

Portions of this report were presented at the annual meeting of the Southwestern Psychological Association, Houston, 1981. Requests for reprints should be directed to the first author at the Department of Psychology, Texas A\&M University, College Station, Texas 77843. The authors wish to thank J. B. Franks and Cheryl Aiken for their technical assistance in carrying out this project. treatment did not induce overt psychomotor arousal, whereas Wellman (unpublished findings) found that a moderate $(20 \mathrm{mg} / \mathrm{kg})$ PPA dosage did not induce hyperthermia in rats. These data tentatively suggest that PPA-induced anorexia is not the result of malaise, yet several tests remain to be completed. Hoebel, Hernandez, and Thompson (1975) noted that a treatment dosage of $40 \mathrm{mg} / \mathrm{kg}$ PPA induced discomfort and general debilitation, but they did not determine whether any dosage of PPA will induce conditioned taste aversion. Experiment 1 therefore provides a dose-response examination of the putative aversive properties of PPA $(10,20$, and $40 \mathrm{mg} / \mathrm{kg})$ as indexed by a conditioned taste aversion paradigm.

\section{EXPERIMENT 1}

\section{Method}

Animals. The animals were $\mathbf{4 0}$ male Sprague-Dawley albino rats weighing $325-440 \mathrm{~g}$ at the beginning of the experiment. The rats were housed individually in standard wire-mesh cages in a temperature-controlled $\left(23^{\circ}-25^{\circ} \mathrm{C}\right)$ colony under a standard light/ dark cycle (lights on at $0800 \mathrm{~h}$ ). A pellet diet (Wayne) was freely available in the home cages throughout the experiment, whereas tap water was available as described in the protocols below.

Drugs. A saline solution consisted of $.9 \%$ sodium chloride dissolved in sterile distilled water. Phenylpropanolamine solutions $(10,20$, and $40 \mathrm{mg} / \mathrm{ml} / \mathrm{kg})$ consisted of phenylpropanolamine hydrochloride (Propadrine; Merck, Sharp, \& Dohme) and sterile distilled water. All PPA solutions were calculated as the salt and were prepared immediately prior to injection.

Procedure. The rats were trained to consume their daily water during a 30-min period on 6 consecutive days. Drinking tests were conducted in separate drinking cages with water, but not food, available in $100-\mathrm{ml}$ graduated Wahman drinking tubes attached 
to the cage front. Fluid consumption was always measured to the nearest $1.0 \mathrm{ml}$. On Day 7, the training day, all rats were presented with a $.1 \%$ saccharin $(w / v)$ solution instead of water during the 30-min drinking period. Immediately after saccharin intake, each rat was injected (ip) with either $.9 \%$ saline $(n=10)$ or 10,20 , or $40 \mathrm{mg} / \mathrm{kg}$ PPA ( $=10 \mathrm{each}$ ) and was returned to the home cage. Days 8-12 constituted the extinction phase of the experiment in which all rats were presented with a two-bottle preference test of water and .1\% saccharin. Bottle position was alternated daily as a control for position preferences.

Statistical analyses. Analyses of variance were made for average group water intake on Days 4-6 prior to drug treatment, for average group saccharin intake on the training day, and for saccharin and water consumption on Days 8-12 (after the latter measures were converted to standard suppression ratios (saccharin/ total fluid intake). Mean differences between groups were evaluated at the .05 level of significance using a priori two-tailed t tests (Kirk, 1968).

\section{Results}

There were no significant between-groups differences either in water intake on Days 4-6 prior to conditioning or in saccharin water intake on Day 7 (Table 1). Figure 1 presents the changes in saccharin intake produced by PPA during Days 8-12. Rats treated with saline on Day 7 displayed a considerable preference for the saccharin solution on Days 8-12, whereas rats treated with the various PPA dosages displayed substantially smaller saccharin intakes. Analyses of variance of the intake data, expressed as suppression ratios, revealed significant main effects of both dosage $[F(3,36)=25.8, p<.001]$ and of day $[F(4,144)=5.6, p<.001]$. The interaction between these factors, however, was not significant $[F(12,144)=1.4, p>.05]$. These analyses indicate that PPA induced substantial dose-dependent taste aversions and that these aversions extinguished at comparable rates over the five extinction periods for each drug group. Further comparisons, using average group daily suppression ratios, indicated that each PPA group displayed significantly smaller suppression ratios than those displayed by saline-treated rats [t $(18)=$ at least $3.3, \mathrm{p}<.005$, for each saline-PPA between-groups comparison].

Informal observation of the rats on Day 7 during a 90-min period following saline and PPA treatment indicated that rats treated with $40 \mathrm{mg} / \mathrm{kg}$ PPA uniformly displayed inhibition of spontaneous motor

Table 1

Average Water Intake on Days $4-6$ and Average Saccharin Intake On Day 7 for Rats Treated With Either Saline or 10,20 , or $40 \mathrm{mg} / \mathrm{kg}$ PPA

\begin{tabular}{lccccc}
\hline & & \multicolumn{4}{c}{ Intake (in Milliliters) } \\
\cline { 3 - 6 } & & \multicolumn{2}{c}{ Water (Days 4-6) } & \multicolumn{2}{c}{ Saccharin (Day 7) } \\
\cline { 3 - 6 } Treatment & $\mathrm{N}$ & Mean & SEM & Mean & SEM \\
\hline Saline & 10 & 17.1 & .52 & 10.8 & 1.3 \\
10 & 10 & 16.6 & .67 & 11.4 & 1.6 \\
20 & 10 & 16.2 & .84 & 10.3 & 1.2 \\
40 & 10 & 16.8 & .50 & 10.6 & 1.2 \\
\hline
\end{tabular}

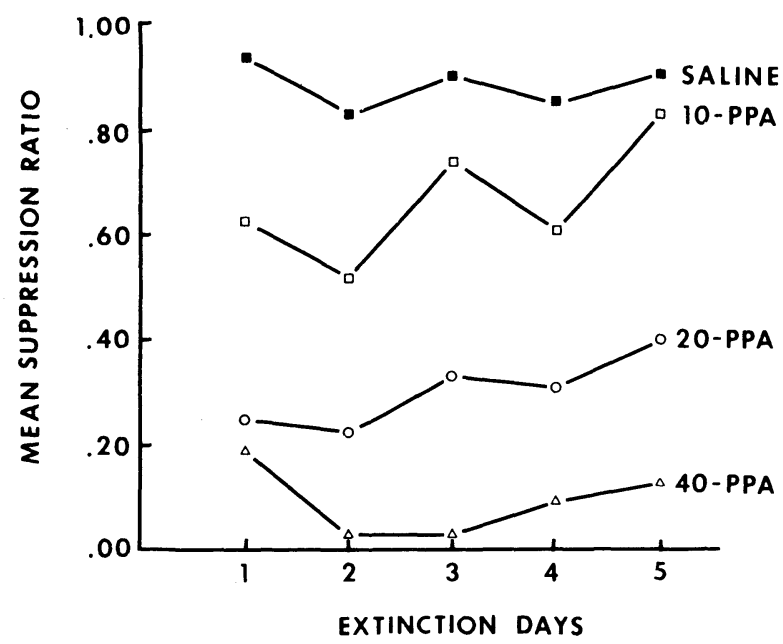

Figure 1. Group mean daily suppression ratios (saccharin/ saccharin plus water) for rats treated with either $.9 \%$ saline or 10 , 20, or $40 \mathrm{mg} / \mathrm{kg}$ phenylpropanolamine (PPA).

movement, piloerection, and a peculiar elevation and rigidity of the tail. These catatonic effects were only infrequently observed in rats treated with either 10 or $20 \mathrm{mg} / \mathrm{kg}$ PPA. Thus, the presence of overt debilitation was not reliably related to the induction of substantial and statistically significant taste aversion by PPA.

\section{Discussion}

Phenylpropanolamine produced substantial conditioned taste aversion at low and moderate dosage levels that did not reliably induce overt changes in ongoing behavior. The magnitude of these aversions was quite unexpected, given previous literature suggesting that PPA is a nontoxic and nonstimulatory drug. Although the data of Experiment 1 would seem to suggest that the anorexia produced by PPA is a consequence of some putative aversive property, another interpretation is possible. Carey and Goodall (1974), working with amphetamine-induced conditioned taste aversion, argued that the primary effect of amphetamine is anorexia and that this anorectic state may be conditioned to a flavor cue. During extinction testing, the flavor presumably elicits a conditioned anorectic state resulting in a decline in the consumption of the drug-paired flavor. Extended to the present data, a conditioned anorexia viewpoint would suggest that the taste aversion to PPA observed in Experiment 1 may simply be a conditioned manifestation of the anorectic property of PPA. A conditioned anorexia viewpoint, however, demands that the anorectic activity of PPA be specific to the ingestion of food (Mueller \& Hsiao, 1977). In support of anorectic specificity for PPA, Hoebel et al. (1975) noted that PPA suppressed feeding elicited by low-level electrical stimulation of lateral hypothalamic (LH) sites but was without influence on 
LH-elicited drinking. The precise relationship between elicited and natural feeding and drinking, however, remains controversial (Valenstein, 1973), and the influence of PPA on ingestive behavior was examined in animals maintained under ad-lib feeding and drinking schedules. Inasmuch as earlier feeding (Kornblith \& Hoebel, 1976; Wellman \& Peters, 1980) and conditioned taste aversion (Experiment 1) testing examined the influence of PPA on consummatory behavior displayed by moderately deprived rats, Experiment 2 sought to determine the specificity of the anorectic properties of PPA by an examination of the influence of PPA $(10,20$, and $40 \mathrm{mg}$ / $\mathrm{kg}$ ) on water intake by 23.5 -h water-deprived rats.

\section{EXPERIMENT 2}

\section{Method}

Animals. The animals were 10 male Sprague-Dawley albino rats weighing $410-470 \mathrm{~g}$ at the beginning of the experiment. The rats were housed and maintained as in Experiment 1.

Procedure. The rats were trained to consume their daily ration of water on seven consecutive daily tests using the intake procedures of Experiment 1. On Days 5-14, water intake was measured after either saline or PPA treatment according to the following protocol: saline (Day 5), saline (Day 6), saline (Day 7), drug (Day 8), saline (Day 9), saline (Day 10), drug (Day 11), saline (Day 12), saline (Day 13), drug (Day 14). Drug and saline treatments were given in a volume of $1.0 \mathrm{ml} / \mathrm{kg}$ and were always given $30 \mathrm{~min}$ prior to each intake test. For the drug tests, each rat received each of the PPA dosages $(10,20$, and $40 \mathrm{mg} / \mathrm{kg}$ ) in a randomized order to minimize possible sequence effects. The saline tests on Days 5-7, 9-10, and 12-13 served to establish that baseline water intake remained stable prior to and during drug testing. For these tests, water (but not food) was available in each test cage and food was freely available in the home cage during each $23.5-\mathrm{h}$ water-deprivation interval.

\section{Results}

One rat died $40 \mathrm{~min}$ after receiving an injection of $40 \mathrm{mg} / \mathrm{kg}$ PPA on Day 8, and the data from this rat were therefore discarded from the experiment. Analysis of water intake after saline treatment on Days $5-7,9-10$, and 12-13 revealed no significant changes in water intake across these days $[F(6,48)=2.1$, $\mathrm{p}>$.10]; these saline values were therefore averaged for each rat, and these average values were used in subsequent one-way analyses of variance of the intake data. Figure 2 presents mean group water intake during a 30-min period following treatment with saline and the various PPA dosages. Analyses of variance revealed that the rats consumed significantly less water after each of the PPA treatments than after injection of saline $[F(3,24)=47.2, p<.001]$. Further between-dosage comparisons revealed that each PPA dosage produced progressively larger suppressions of water intake $[\mathrm{t}(24)=$ at least 4.2 for each comparison, $\mathrm{p}<.001]$. The present data therefore demonstrate that PPA induces a dose-dependent suppression of water intake. Finally, the $40-\mathrm{mg} / \mathrm{kg}$ treatment reliably induced the catatonic effects noted

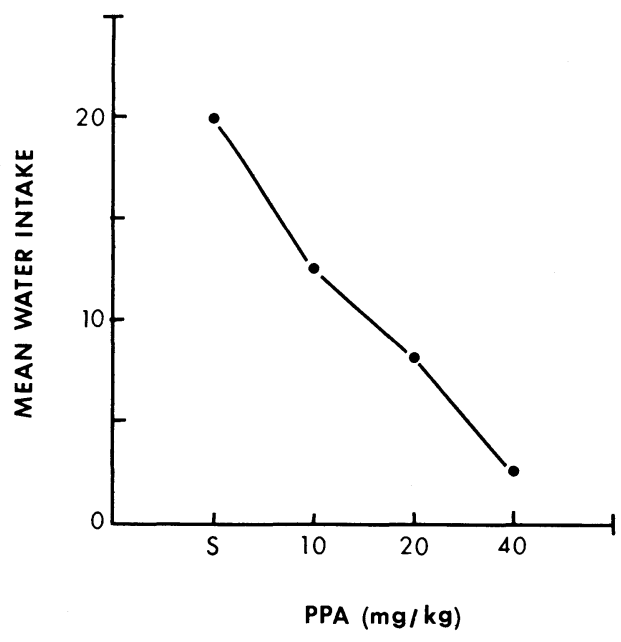

Figure 2. Group mean 30-min water intake (mls) for rats treated with saline (S) and with 10,20 , and $40 \mathrm{mg} / \mathrm{kg}$ phenylpropanolamine.

in Experiment 1, whereas the 10- and 20-mg/ $\mathrm{kg}$ PPA treatments did not.

\section{GENERAL DISCUSSION}

Gibbs and Smith (1977) provided a cogent argument that the observation of conditioned taste aversion to an anorectic drug does not necessarily imply that anorexia is a consequence of some malaise property of the drug in that the threshold for the anorectic activity may lie well below the threshold for taste aversion. Although the latter notion is supported by the observation of significant anorexia to a PPA dosage of $5 \mathrm{mg} / \mathrm{kg}$ (Kornblith \& Hoebel, 1976), approximate equivalence of dose-response curves for the anorexia induced by PPA and for the taste aversion induced by PPA has also been observed (Wellman $\&$ Peters, 1980, Experiment 1). Moreover, the dosedependent suppression of water intake to PPA observed in Experiment 2 demonstrates that the anorectic activity of PPA is nonspecific and indirectly suggests that the anorexia induced by PPA may be a consequence of some aversive action of the drug. Although direct statistical comparisons of the percent changes from saline baseline for conditioned taste aversion (Experiment 1) and for hypodipsia (Experiment 2) are not permissible, it is interesting to note that the dose-response curves for these effects of PPA were comparable. The similarity of the magnitude of these changes indicates that a single property of PPA, presumably malaise, produced these effects. Although these data provide strong support for the contention that moderate anorectic activity of PPA is secondary to malaise, a final conclusion awaits the demonstration that PPA acts as a negative reinforcer in a self-administration paradigm. To the extent that PPA is truly aversive, animals should 
respond to terminate programmed intravenous administration of PPA (Hoffmeister, 1975) and should not emit operant responses that result in intravenous PPA administration (Pickens \& Harriss, 1968).

Abdallah (1968) and, more recently, Hoebel (1976) suggested that PPA may induce anorexia via a CNS satiety mechanism. Hoebel (1976) suggested that lateral hypothalamic (LH) sites that support selfstimulation and yield elicited feeding and drinking are apparently under the control of the nutritional status of the animal. Manipulations that increase food motivation such as deprivation or insulin produced an enhancement of self-stimulation (SS) and a decline in stimulation-escape (i.e., SE, the responding of an animal to terminate programmed LH stimulation). Conversely, manipulations that decrease food motivation, such as induced obesity or gastric loading, produced decreased SS and enhanced SE (Balagura \& Hoebel, 1967; Hoebel \& Thomas, 1969). In support of a CNS satiety notion, Hoebel et al. (1975) reported data suggesting that PPA produced a satiety pattern of responding; that is, PPA treatment produced decreased SS and enhanced SE.

Although this paradigm is reported to be uniquely sensitive to the appetitive motivational state of an animal, it is clear that other factors, unrelated to feeding, may produce changes in responding in the SS and SE paradigm. Amphetamine, a stimulant, produced enhancement of both SS and SE, whereas fenfluramine, a sedative, produced a decline in both SS and SE; both drugs, however, have anorectic properties (Kornblith \& Hoebel, 1976). Moreover, several of the manipulations reported to alter food motivation and rates of SS and SE are known to induce malaise: gastric loading, for example, induces taste aversion (Deutsch, Molina, \& Puerto, 1976). Thus, manipulations such as gastric loading or PPA may induce a moderate level of malaise sufficient to induce a decline in self-stimulation, and this malaise state may summate with the well-known aversive state associated with programmed stimulation of the lateral hypothalamus (Olds \& Olds, 1963; Roberts, 1958). The elevated stimulation-escape responding observed after PPA treatment may reflect an attempt to terminate at least that portion of the aversive state that is induced by forced LH stimulation. Moreover, a central action of PPA is precluded by data from Axelrod (1953) and from Thiercelin, Jacquot, Rapin, and Cohen (1976) demonstrating minimal uptake of PPA by the brain over a 60-min period following peripheral administration. By way of contrast, the anorectic effects of PPA on food and water intake are apparent at 30 min posttreatment. Thus, the available data indicate that PPA, presumably via a peripheral mechanism, induces anorexia for both food and water intake and induces conditioned taste aversion, and that the effects of PPA on hypothalamic self-stimulation and stimulation-escape may be interpreted in terms of the putative peripheral aversive properties of PPA.

\section{REFERENCES}

Abdallah, A. H. Anorectic activity of ephedrine isomers. Life Sciences, 1908, 7, 665-670.

Aviado, D. M. Sympathomimetic drugs. Springfield, Ill: Thomas, 1970.

Axelrod, J. Studies on sympathomimetic amines. Journal of Pharmacology and Experimental Therapeutics, 1953, 109, 62-73.

Balagura, S., \& Hoebel, B. G. Self-stimulation of the lateral hypothalamus modified by insulin and by glucagon. Physiology \& Behavior, 1967, 2, 337-340.

CAREY, R. J. Facilitation of responding for rewarding brain stimulation by a high dose of amphetamine when hyperthermia is prevented. Psychopharmacology, 1979, 61, 267-271.

Carey, R. J., \& Goodall, E. B. Amphetamine-induced taste aversion: A comparison of d- versus 1-amphetamine. Pharmacology, Biochemistry and Behavior, 1974, 2, 325-330.

Carlton, P. L. Cholinergic mechanisms in the control of behavior. Psychological Review, 1963, 70, 19-39.

Costa, E., \& Garattini, S. Amphetamines and related compounds. New York: Raven Press, 1970.

Deutsch, J. A., Molina, F., \& Puerto, A. Conditioned taste aversion caused by palatable nontoxic nutrients. Behavioral Biology, 1976, 16, 161-174.

Epstein, A. E. Suppression of eating and drinking by amphetamine and other drugs in normal and hyperphagic rats. Journal of Comparative and Physiological Psychology, 1959, 52, 37-45.

GibBs, J., \& SмIтн, G. P. Gibbs and Smith reply. Nature, 1977, 266, 196.

Griboff, S. I., Berman, R., \& Silverman, H. I. A doubleblind clinical examination of a phenylpropanolamine-caffeinevitamin combination and a placebo in the treatment of exogenous obesity. Current Therapeutic Research, 1975, 17, 535-543.

Hoebel, B. G. Satiety: Hypothalamic stimulation, anorectic drugs, and neurochemical substrates. In D. Novin, W. Wyrwicka, \& G. Bray (Eds.), Hunger: Basic mechanisms and clinical implications. New York: Raven Press, 1976.

Hoebel, B. G., Hernandez, L., \& Thompson, R. P. Phenylpropanolamine inhibits feeding, but not drinking induced by hypothalamic stimulation. Journal of Comparative and Physiological Psychology, 1975, 89, 1046-1052.

Hoebel, B. G., \& Thomas, R. D. Aversion to lateral hypothalamic stimulation caused by intragastric feeding or obesity. Journal of Comparative and Physiological Psychology, 1969, 68, 536-543.

HoFFMEISTER, F. Negative reinforcing properties of some psychotropic drugs in drug-naive rhesus monkeys. Journal of Pharmacology and Experimental Therapeutics, 1975, 192, 468-477.

KIrK, R. E. Experimental design: Procedures for the behavioral sciences. Belmont, Calif: Brooks/Cole, 1968.

Kornblith, C. L., \& Hoebel, B. G. A dose response study of anorectic drug effects on food intake, self-stimulation, and stimulation-escape. Pharmacology, Biochemistry and Behavior, 1976, 5, 215-218.

Mueller, K., \& Hsino, S. Specificity of cholecystokinin satiety effect: Reduction of food but not water intake. Pharmacology, Biochemistry and Behavior, 1977, 6, 643-646.

OLDs, M. E., \& OLDs, J. Approach-avoidance analysis of rat diencephalon. Journal of Comparative Neurology, 1963, 120, 259-295.

Pickens, R., \& Harriss, W. C. Self-administration of d-amphetamine by rats. Psychopharmacologia, 1968, 12, 158-163.

RoBERTs, W. W. Both rewarding and punishing effects from 
stimulation of posterior hypothalamus of cat with same electrode at same intensity. Journal of Comparative and Physiological Psychology, 1958, 51, 400-407.

Thiercelin, J. F., Jacquot, C., Rapin, J. R., \& Cohen, Y. Pharmacokinetics of 14C-DL-norephedrine in the rat. Archives of International Pharmacodynamics and Therapeutics, 1976, 220, 153-163.

VAlenstein, E. S. Brain stimulation and motivation. Glenview, Ill: Scott, Foresman, 1973.
Wellman, P. J., \& Peters, R. H. Effects of amphetamine and phenylpropanolamine on food intake in rats with ventromedial hypothalamic on dorsolateral hypothalamic damage. Physiology \& Behavior, 1980, 25, 819-827.

(Received for publication March 6, 1981; revision accepted April 17, 1981.) 